\title{
Advanced Panspermia
}

\section{Wojciech Konrad Kulczyk ${ }^{*}$}

The New Genesis Foundation, Camberley, Surrey, UK

*Corresponding author: Wojciech Konrad Kulczyk, Camberley, Surrey, UK, Tel: 44 7780796032; E-mail: konrad3@btinternet.com

Received date: May 16, 2017; Accepted date: June 8, 2017; Published date: June 13, 2017

Copyright: ( 2017 Kulczyk WK. This is an open-access article distributed under the terms of the Creative Commons Attribution License, which permits unrestricted use, distribution, and reproduction in any medium, provided the original author and source are credited.

\begin{abstract}
In this study a new hypothesis of advanced panspermia is proposed. The theory of panspermia is the most plausible hypothesis for explaining the origins of life on Earth and is supported by ample evidence. The current hypothesis of panspermia doesn't explain two critical milestones in the development of advanced life on Earth: the Cambrian explosion and the human brain. The Cambrian explosion saw the almost simultaneous arrival of the major body plans of all existing animals. During this time the complexity of life increased by several orders of magnitude. The second milestone also marked an enormous increase in the complexity of life. The human brain is far more complex than the animal brain and its development took place over several stages. Several hominid groups existed during the last few million years but show no link to Homo sapiens. The genetic make-up of Neanderthals, our closest cousins, is so different from ours that our descent from them can be excluded. The proposed process of advanced panspermia explains the mechanism of these two milestones. It includes the sending of animal embryos to Earth and the genetic modification of the human embryo.
\end{abstract}

Keywords: Panspermia; Extra-terrestrial; Origin of life; Evolution; Cambrian explosion; Human brain; Abiogenesis; SETI; Homo sapiens, Neanderthals

\section{Introduction}

Abiogenesis is the dominating theory which explains the origin of life on Earth. It evolved from the primordial soup theory to the present-day RNA World hypothesis. It is possible that it could be shown that some components of life could have originated from inorganic matter but proving their transition to the living cell would be much more difficult.

There are two problems facing abiogenesis. The first problem is that the cell architecture is not coded by DNA. This means that genes provide information on how to build basic blocks of life such as proteins, enzymes, molecular complexes, etc., but that genomes contain no genes that specify cellular forms such as membranes or cytoskeletons [1]. So genes specify molecular parts, but not their arrangement into a higher order. This means that every cell originates from a mother cell.

The second problem is that even the simplest cell has a sophisticated control system which drives the cell's life cycle [2]. The cell cycle system is comprised of multiple modular subsystems that implement cellular growth and reproduction. An integral control system, constructed using biochemical and genetic logic circuitry, organizes the timing of initiation of each of these modular functions, similar to electronic control systems designed by man. This control system is not coded by the cell DNA but is inherited from the mother cell. Since genes are not involved with the cell structure and its control system, it is difficult to envisage how abiogenesis could be responsible for the origins of life.

These problems are not applicable to the panspermia hypothesis which was revived by Crick and Orgel [3]. Hoyle and Wickramasinghe [4] confirmed that life could have come from the cosmos and further research shows that bacteria and even higher organisms could have survived a journey through space. Since these proposals, molecular and genetic research has shown that life is far more complex than was ever suspected and panspermia is becoming a serious alternative to the theory of evolution.

The fact that life started very soon after Earth became habitable is one of the strongest arguments for panspermia. It is assumed that Latest Universal Common Ancestor, LUCA, which existed about 4 billion years ago, was a very sophisticated organism having between 500 and 1000 genes and fully developed photosynthesis and respiration systems. Therefore, there would not have been enough time for LUCA to evolve from the considered progenitor of life.

After the seeding of Earth, it was assumed that life developed further as a result of evolution. Since it is doubtful that Darwinian evolution was responsible for the generation of complex organisms which needed thousands of beneficial mutations, it was proposed that strong panspermia [5] could be responsible for further development. This hypothesis postulates that new genes from space sustained evolutionary progress. It is known that horizontal gene transfer in bacteria modifies the genetic makeup of organisms. It is possible that viruses from space could have injected their genetic material into bacteria and modified their genes. It has been proposed that panspermia could be intentional, although some believe that it was completely fortuitous.

Panspermia provides plausible explanations for the origin of life and the arising of eukaryotic cells on Earth. However, there are two milestones in the development cycle of intelligent life that might be difficult to explain using simple panspermia. These two milestones are: the Cambrian explosion and the human brain.

\section{Cambrian Explosion}

During the Cambrian explosion, around 540 million years ago, the body plans of all existing phyla belonging to the animal kingdom suddenly appeared. During the 20 million year period there appeared such different classes of animals as vertebrates [6], arachnids and 
molluscs. These animals did not arrive in sequence from simple to more complex forms, but turned up almost simultaneously.

Most significantly, during this period the complexity of organisms increased by several orders of magnitude. In comparison with eukaryotic cells, new organisms had tens of thousands of new proteins and their genome was considerably increased to approximately 20,000 genes.

Even the simplest animals needed new organs such as skin, a skeleton, muscles, neurons, and digestive, reproductive, respiratory and circulation systems. Besides these organs, more advanced animals had eyes, a brain, a spine and various sensors. To build these organs, approximately 200 different types of cells were used.

To get a glimpse of how complex the building process of new organisms is, we have to look at embryo development. The whole process is controlled by Hox genes [7]. These genes provide the overall layout of the body, defining the position of the main parts such as the head, trunk, limbs, internal organs, etc. Each of the Hox genes switches on other genes which provide more detailed information about the position and structure of different tissues needed to make these parts. Eventually the position and type of almost every cell in the body must be specified. If we consider that organisms consist of trillions of cells, the embryo development control system is extremely complex, and has been since the earliest animals.

The complexity of embryo assembly can be seen in the example of the human eye which is controlled by about 2,000 genes. In the human embryo, approximately 3,000 gene regulatory compounds are at work. The embryo development control system had to have been introduced as a complete unit because even small genetic deviations would have resulted in detrimental results.

It is highly unlikely that the simultaneous development of large morphological structures and complex genetic control systems arose as a result of implanting a few new genes. Therefore, the mechanism of simple panspermia could not have been responsible for such developments.

\section{Homo sapiens}

There is extensive scientific evidence showing that Homo sapiens suddenly appeared in Africa about 200,000 years ago. The dating of this event is supported by the earliest human fossils from this period and the mitochondrial DNA inherited through the maternal line. Modern people's mitochondrial DNA can all be traced back to a common ancestor, an 'Eve' that lived about 200,000 years ago.

Neanderthals, which appeared possibly about 600,000 years ago, were our closest relatives. However vast numbers of Neanderthal genes are not carried by contemporary humans [8]. Results from new studies confirm that Neanderthal and human genomes are more than $99.5 \%$ identical, differing by about 3 million bases. The Neanderthal mitochondrial DNA sequences are substantially different from modern human mtDNA [9]. Researchers compared the Neanderthal, modern human and chimpanzee sequences. Most human sequences differ from each other by on average 8.0 substitutions, while the human and chimpanzee sequences differ by about 55.0 substitutions. The Neanderthal and modern human sequences differ by approximately 27.2 substitutions. This would indicate a significant separation between these two groups.

Genetic studies show that $H$. sapiens did not descend from Neanderthals and we separated from them about 400,000 years ago. It is assumed that Homo heidelbergensis that lived in Africa, Europe and western Asia between 600,000 and 250,000 years ago is the ancestor to not only Neanderthals but also $H$. sapiens, but there is no genetic evidence to confirm this. $H$. heidelbergensis looked very similar to Neanderthals but was significantly different from $H$. sapiens.

Therefore, one could say that there is no direct link between $H$. sapiens and any prior hominid group [10]. Because there is no indication who our direct ancestors were, a hypothesis was proposed that $H$. sapiens continuously evolved over several hundred thousand years [11]. However, there is no fossil evidence supporting this hypothesis.

An extensive list of tools used by humanoid species, which were dated from several millions of years ago, is provided by Cremo and Thompson [12]. This shows that the origin of humanoid species is much older than recognised by mainstream academia and there is no clear line of progression from less developed to more advanced hominids.

What distinguishes us from the animal world is our unique brain. The functioning of the human brain is an extension of the operation of the animal brain which has been developed since the Cambrian explosion. The human brain has several functions common to mammals such as vision, hearing and positioning system [13]. However, the human mind has several very special properties such as consciousness and logical and abstract thinking which distinguish it from the animal brain.

The genetic development of the human brain is full of unknown stages. It appears that at least a third of approximately 20,000 different genes that make up the human genome are primarily active in the brain. This means that the brain has the most complex structure of the entire body. There are two clusters of hundreds of individual genes that are thought to influence all our cognitive functions: including memory, attention, processing speed and reasoning.

The genetic study of Neanderthals shows that, in genes responsible for making proteins, 87 differ from those found in modern humans [14]. Some gene differences are involved in both the immune responses and the development of brain cells. One can assume that $H$. heidelbergensis had a less developed brain than Neanderthal man, therefore there must be an even bigger difference between the brains of $H$. heidelbergensis and H. sapiens.

It was shown by the Lahn group at the Howard Hughes Medical Institute at the University of Chicago that further modification of the human brain took place about 37,000 years ago when the prevalent microcephalin variant [15] emerged, while the dominant ASPM variant appeared about 5,800 years ago [16].

\section{Advanced panspermia}

The question arises whether strong panspermia could be responsible for the development of life during and after the Cambrian explosion. Our understanding of embryo development is that thousands of new genes and gene control substances were involved in the development of higher organisms. Also, it is difficult to envisage the partial development of the pyramidal structure of the Hox gene control system and this indicates that the architecture of the control system would have to have been developed as a complete operational unit. It looks as though such a system would not have been able to be generated by evolution or strong panspermia. 
To explain the development of complex life on Earth we have to consider that a different mechanism was in operation, and I call this mechanism advanced panspermia. It is possible that intelligent life on Earth is the result of a purposeful experiment carried out by an extraterrestrial civilization. This experiment would have involved seeding Earth with the first cells to prepare the Earth's biosphere for supporting more complex life. Once Earth had a sufficient amount of oxygen, multicellular life was introduced during the Cambrian explosion.

It is possible that the Cambrian explosion started when the embryos of all phyla were sent in special containers suitable for space travelling. Since all life was water based, such embryos placed in water or ice would be protected against radiation. One could envisage that the ocean fauna was prepared in such a way, that simple organisms were sent first, followed by more advanced animals.

Such transport could have been used in the further development of water based animals but would not be suitable for land based animal embryos. The development of land based animals would have taken place via genetic modifications of germ cells, which are normally placed inside animal bodies. Such modifications could have been achieved by internal genetic variability mechanisms which exist in chromosomes or by suitably engineered viruses. It is possible that transposons played a significant role in this development process [17].

How could the development of $H$. sapiens proceed? It is possible that the development of early hominids progressed using specially designed viruses or other genetic modifications. Tattersall believes that at least 20 hominid species existed during the last several million years. He says: "Our biological history has been one of sporadic events rather than gradual accretions. Over the past five million years, new hominid species have regularly emerged, competed, coexisted, colonized new environments and succeeded or failed" [18]. This process produced many different groups of apelike and humanlike beings. According to Cremo and Thompson, there is overwhelming evidence that advanced human forms were already present several million years ago [12].

The existence of so many hominid species which disappeared without a trace indicates that the brain modification methods used were not very successful. The problem was that while the upright body was easily implemented, the development of the brain was much more difficult. Brain modification requiring changes to hundreds of genes needed very precise genetic manipulations.

This problem can be better understood when we look at the development of Neanderthals. The Neanderthals had a very large brain [19], about 1,600 cubic centimetres, but still its performance was far from what was needed. The problem was that the new hominid groups arising from the old groups carried many old genes. These genes impeded brain development but they could not be easily removed. This is confirmed by the study of the Neanderthal genome. There is a strong indication that the Neanderthal's genes were harmful to humanNeanderthal hybrids and their descendants [20].

A possible solution was to use direct genetic modifications. The proposed scenario is that the $H$. sapiens embryo was produced by in vitro fertilization and was implanted in a suitable surrogate mother. In that way, it was guaranteed that new genes were introduced and all unwanted or harmful genes were removed or modified.

One cannot exclude the possibility that the surrogate mother was a Neanderthal woman, therefore early $H$. sapiens could have lived together with Neanderthals. This could explain why about $2 \%$ of the
DNA of people with European ancestry can be traced to Neanderthals, while some other ethnic groups can have up to $6 \%$ of Neanderthal genes. Several genetic studies indicate that there is a vast genetic difference between H. sapiens and Neanderthals [14]. Such a big difference could only arise in such a short period of time as the result of purposeful genetic modifications.

The hypothesis of the involvement of extraterrestrial civilizations in the arising of intelligent life on Earth should be considered because the existence of such civilizations is an accepted hypothesis as shown by the SETI programme which searches for alien signals. This search has not brought any results because we assume that these civilizations are similar or only slightly more advanced than ours. However, it is not unrealistic to assume that extraterrestrial civilizations could be several million or maybe even billions of years old, therefore their capabilities would be much more advanced than ours making them very difficult to contact.

Support for extraterrestrial design also comes from the analysis of our genetic code. Work performed by Shcherback and Makukov shows unexplained properties of DNA codes which would require some intelligent approach that cannot be accounted for by Darwinian evolution. They say that "simple arrangement of the code reveals an ensemble of arithmetical and ideographical patterns of symbolic language. Accurate and systematic, these underlying patterns appear as a product of precision logic and nontrivial computing rather than of stochastic processes" [21]

A particular property of the human brain which could not have arisen as a result of simple genetic modifications is its ability for further development. This development is most visible in the domain of consciousness. It is known that the genetic makeup of the human brain has not changed for the last 6000 years, but we observe significant development of human thinking and consciousness, for example concern about the environment, climate change and the Earth's future. This shows that the human brain has built in a function enabling further development which is not based on genetic or evolutionary mechanisms. Therefore one could predict that the development of the human race will result in a more humanitarian society. Looking at the present state of the world we might be under the impression that things are getting worse. However, if we compare our present situation with the situation a hundred years ago we can see tremendous progress in practically all aspects of our lives.

There is a growing body of evidence showing that life on Earth is a far more complex phenomenon than we have ever considered it to be. The most complex organ without any doubt is the human brain. It is very likely that extraterrestrial beings were responsible for its design. However, implementing this design in human beings would require the direct contact of extraterrestrials with Earth. It is difficult and maybe unwise to speculate about the extraterrestrial civilization. Such a civilization would have had to exist for at least 4 billion years and therefore be advanced beyond our comprehension.

\section{References}

1. Harold FM (2005) Molecules into cells: specifying spatial architecture. Microbiol Mol Biol Rev 69: 544-564.

2. McAdams HH, Shapiro L (2009) System-level design of bacterial cell cycle control. FEBS Lett 583: 3984-3991.

3. Crick FHC, Orgel LE (1973) Directed panspermia. Icarus 19: 341-346.

4. Hoyle F, Wickramasinghe NC (1980) Evolution from space. JM Dent, London. 
5. Klyce B (2001) Panspermia Asks New Questions. The Search for Extraterrestrial Intelligence (SETI) in the Optical Spectrum- Conference 22-24 January 2001, San Jose CA: SPIE Proceedings-4273, pp: 11-14.

6. Morris SC, Caron JB (2014) A primitive fish from the Cambrian of North America. Nature 512: 419-422.

7. Mallo M, Alonso CR (2013) The regulation of Hox gene expression during animal development. Development 140: 3951-3963.

8. Sankararaman S, Patterson N, Li H, Pääbo S, Reich D (2012) The date of interbreeding between neandertals and modern humans. PLOS Genetics 8: $1-9$.

9. Krings M, Stone A, Schmitz RW, Krainitzki H, Stoneking M, et al. (1997) Neandertal DNA sequences and the origin of modern humans. Cell 90: 19-30.

10. Kennedy G (1980) The emergence of modern man. Nature 284: 11-12

11. Weaver TD (2012) Did a discrete event 200,000-100,000 years ago produce modern humans? J Human Evol 63: 121-126.

12. Cremo MA, Thompson RL (1999) The hidden history of the Human Race. Bhaktivedanta book publishing, Los Angeles.

13. Moser EI, Roudi Y, Witter MP, Kentros C, Bonhoeffer T, et al. (2014) Grid cells and cortical representation. Nat Rev Neurosci 15: 466-481.
14. Prüfer K, Racimo F, Patterson N, Jay F, Sankararaman S, et al. (2014) The complete genome sequence of a Neanderthal from the Altai Mountains. Nature 505: 43-49.

15. Evans PD, Gilbert SL, Mekel-Bobrov N, Vallender EJ, Anderson JR, et al. (2005) Microcephalin, a gene regulating Brain size, continues to evolve adaptively in humans. Science 309: 1717-1720.

16. Mekel-Bobrov N, Gilbert SL, Evans PD, Vallender EJ, Anderson JR, et al (2005) Ongoing adaptive evolution of ASPM, a Brain size determinant in Homo sapiens. Science 309: 1720-172.

17. Feschotte C, Pritham EJ (2007) DNA transposons and the evolution of eukaryotic genomes. Ann Rev Genet 41: 331-368.

18. Tattersall I (2000) Once We Were Not Alone. Sci Am 282: 56-62.

19. Clinton K (2015) Average Cranium/ Brain size of Homo neanderthalensis vs. Homo sapiens. The Backbone.

20. Juric I, Aeschbacher S, Coop G (2016) The strength of selection against Neanderthal introgression. PLOS Genet.

21. Shcherbak VI, Makukov MA (2013) The "Wow! signal" of the terrestrial genetic code. Icarus 224: 228-242. 